\title{
Feedback Control Over Lossy SNR-Limited Channels: Linear Encoder-Decoder-Controller Design
}

\author{
Subhrakanti Dey, Senior Member, IEEE, Alessandro Chiuso, Senior Member, IEEE, \\ and Luca Schenato, Senior Member, IEEE
}

\begin{abstract}
In this paper, we consider the problem of encoding and decoding codesign for linear feedback control of a scalar, possibly unstable, stochastic linear system when the sensed signal is to be transmitted over a finite capacity communication channel. In particular, we consider a limited capacity channel which transmits quantized data and is subject to packet losses. We first characterize the optimal strategy when perfect channel feedback is available, i.e., the transmitter has perfect knowledge of the packet loss history. This optimal scheme, innovation forwarding hereafter, is reminiscent of differential pulse-code modulation schemes adapted to deal with state space models, and is strictly better than a scheme which simply transmits the measured data, called measurement forwarding (MF) hereafter. Comparison in terms of control cost as well as of critical regimes, i.e., regimes where the cost is not finite, are provided. We also consider and compare two popular suboptimal schemes from the existing literature, based on 1) state estimate forwarding and 2) measurement forwarding, which ignore quantization effects in the associated estimator and controller design. In particular, it is shown that surprisingly the suboptimal MF strategy is always better then the suboptimal state forwarding strategy for small signal-to-quantization-noise-ratios.
\end{abstract}

Index Terms-Control under communication constraints, linear quadratic Gaussian (LQG) control, packet losses, quantization.

\section{INTRODUCTION}

The interplay between control stability and nonidealities of communication channels has attracted considerable attention in the past decade, mainly driven by the success of wireless communication and its penetration into automation and control applications. From a theoretical perspective, we have witnessed the convergence of control theory, communication theory, and information theory which have led to remarkable and interesting results in terms of the ultimate performance limitations which take into account both the dynamical systems characteristic, typically their unstable eigenvalues and nonminimum phase zeros, and the channel characteristic, typically its capacity [1]-[11].

Nonetheless, there are still important open questions that need to be answered. For example, most of the results are obtained for scenarios

Manuscript received April 21, 2016; revised October 31, 2016; accepted February 11, 2017. Date of publication February 23, 2017; date of current version May 25, 2017. This work was supported by the European Community's Seventh Framework Programme [FP7/2007-2013] under Grant Agreement 257462 HYCON2 Network of excellence and by the Swedish Research Council under Grant DNR 621-2013-5395. Recommended by Associate Editor S. Andersson.

S. Dey is with the Department of Signals \& Systems, Uppsala University, Uppsala, SE 75121, Sweden (e-mail: Subhra.Dey@ @ignal.uu.se).

A. Chiuso and L. Schenato are in the Department of Information Engineering, University of Padova, Padova 35131, Italy (e-mail: chiuso@dei.unipd.it; schenato @ dei.unipd.it).

Color versions of one or more of the figures in this paper are available online at http://ieeexplore.ieee.org

Digital Object Identifier 10.1109/TAC.2017.2674024 in which the transmitter has full knowledge of what happened over the channel in the past (perfect channel feedback), which guarantees several separation principles both in terms of controller/observer design and of source/channel coding, see e.g., [12] for early references. Differently, if the channel feedback is not present or it is "imperfect," very few results are available and mainly based on heuristics [13]-[18]. Another difficulty arises when both packet loss and quantization are considered simultaneously. In fact, if either of these two limitations is neglected, the resulting optimal strategies can be quite different. For example, in a scenario with packet loss only, Gupta et al. [19] showed that the optimal strategy is to send the estimate of the state over the channel, and it is therefore quite attractive. Differently, if only rate limitation, i.e., the maximum number of symbols that can be transmitted per unit of time, is considered, then differential coding results to be the optimal strategy as in differential pulse-code modulation (DPCM) [20]. Obviously, this strategy is rather different from the one that transmits the complete state estimate. Recently, in [21] we have shown that a strategy inspired by DPCM is optimal in the context of remote estimation when considering both packet loss and quantization limitations for a scalar stable system when full channel feedback is present. However, the optimal strategy in the presence of imperfect channel feedback remains elusive and only some sensible heuristic schemes have been proposed in [21]. In the context of closed loop unstable control system, simultaneous analysis of packet loss and quantization has been studied in [11] assuming that the transmitter simply forwards a quantized version of the raw measurement (measurement forwarding). Another related work is [22] which obtains minimum data rates for mean square stabilizability of linear systems over lossy channels. However, unlike [22], the focus of this paper is not just stabilizability, but linear optimal encoder-decoder-controller design for optimizing the control performance.

In this paper, expanding on [18], we extend the results of [21] and [11] by also including the encoder design (as in [21]) in the control problem considered in [11]. We show that the optimal strategy when full channel feedback is available at the transmitter is to send the difference from the estimated state at the transmitter and the predicted state at the receiver [(innovation forwarding (IF)] as in [21] followed by a constant gain (as in [11]) state feedback, where the receiver state estimate is provided by a Kalman filter. However, although the stability region remains the same, the control performance is improved as compared to the strategy proposed in [11]. We also propose two suboptimal strategies which neglect the presence of quantization, based on state forwarding (SFs) proposed in [19] and measurement forwarding (MFs) proposed in [5]; the "s" in "SFs" and "MFs" stands for suboptimal. We analyze the stability regions of these schemes and compare their performance with the optimal IF and MF strategies. In particular, it is shown than none of these two suboptimal strategies is superior to the other in all regimes; however, quite surprisingly, MFs is always better than SFs for small signal-to-quantization-noise-ratio (SQNR). 


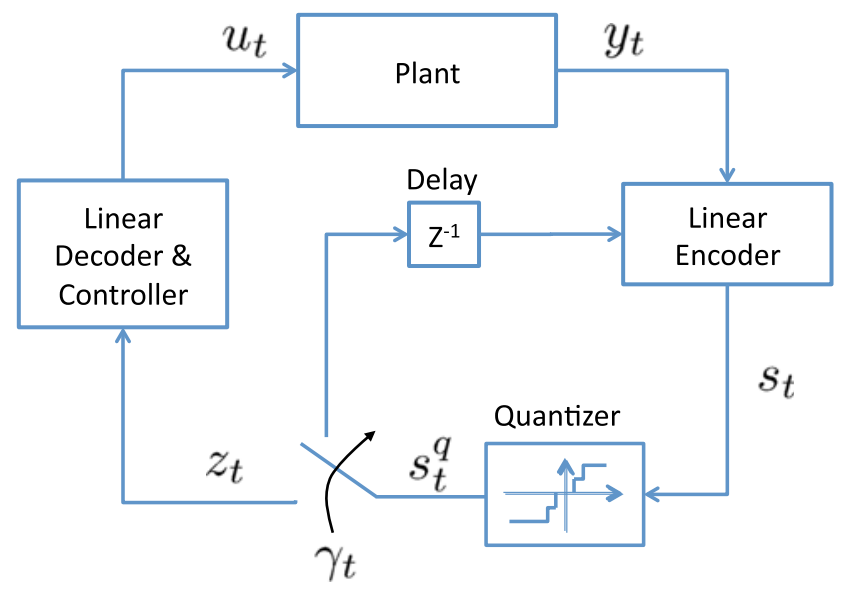

Fig. 1. General scheme of the control system under communication constraints. The sequence $\gamma_{t}$ is fed back to the coder only when channel feedback is available.

\section{SyStem MOdel AND PROBLEM Formulation}

We consider a discrete-time linear scalar time-invariant possibly unstable $^{1}$ plant $(|a| \geq 1)$ as given below:

$$
\begin{aligned}
x_{t+1} & =a x_{t}+b u_{t}+w_{t}, \quad b=1 \\
y_{t} & =x_{t}+v_{t}
\end{aligned}
$$

where $w_{t}, v_{t}$ are the process and measurement noise assumed to be independent and identically Gaussian distributed with zero mean and variances $\sigma_{w}^{2}$, and $\sigma_{v}^{2}$, respectively. To simplify the notation, w.l.o.g. the scalar parameter $b$ is set to one. In the networked control scenario we consider, the sensor output $y_{t}$ may be encoded to generate a signal $s_{t}$ which is transmitted to a remote estimator/controller over a communication channel. We restrict $s_{t}$ to be a causal linear function of the measurements $\mathcal{Y}_{t} \triangleq\left\{y_{0}, y_{1}, \ldots, y_{t}\right\}$. The linear controller produces a control input $u_{t}$, see Fig. 1. We shall prove that the optimal controller will be the cascade of a Kalman estimator followed by a constant gain state-feedback controller. The objective of the control design problem is to find optimal $\left\{s_{t}\right\}$ and $\left\{u_{t}\right\}$ such that the average LQ cost

$$
J_{\infty}=\lim _{T \rightarrow \infty} \frac{1}{T} \sum_{t=0}^{T-1}\left(\mathbb{E}\left[y_{t}^{2}\right]+\rho \mathbb{E}\left[u_{t}^{2}\right]\right)
$$

where $\rho \geq 0$ is (finite and) minimized. We assume that the channel between the sensor (which senses the plant output) and the remote estimator/controller is subject to both bandwidth constraints and packet losses. Thus, the encoded signal $s_{t}$ is quantized using a finite number of bits, producing $s_{t}^{q}$ which is to be transmitted. Under a fine quantization assumption, it has been established by multiple authors [23]-[25] that the quantization error $\tilde{s}_{t}^{q}:=s_{t}^{q}-s_{t}$ can be well approximated by an additive white Gaussian noise (AWGN) independent of $s_{t}$, with a variance that is proportional to $\operatorname{Var}\left\{s_{t}\right\}$. If fact, it has been shown in [21] that a uniform scalar quantizer with as few as 3-4 bits per sample provides results that are sufficiently close to the theoretical values based on the AWGN model used also this work. Thus, the quantized signal can be written as $s_{t}^{q}=s_{t}+n_{t}$, where $n_{t}$ is AWGN with zero mean and variance $\frac{\mathbb{E}\left[s_{t}^{2}\right]}{\Lambda}$, where $\Lambda$ is known as the signal-to-quantization noise ratio (SQNR).

\footnotetext{
${ }^{1}$ When the plant is stable $(|a|<1)$ there is no issue of stabilizability, so that the cost (2) is finite also without feedback control (e.g., with $u_{t}=0$ ); thus, no requirement needs to be made on the SQNR and the packet loss probability. Note however that even if $|a|<1$ it still makes perfect sense to optimize (2).
}

The received signal $z_{t}$ (see Fig. 1) is modeled by the multiplicative equation $z_{t}:=\gamma_{t} s_{t}^{q}$ where the packet loss process $\gamma_{t} \in\{0,1\}$ is assumed to be an independent and identically distributed (i.i.d.) Bernoulli process with packet loss probability $P\left(\gamma_{t}=0\right)=\epsilon$. When $\gamma_{t}=1$, $z_{t}=s_{t}^{q}$. On the other hand when $\gamma_{t}=0$, the transmitted information is lost. For optimal linear encoder/decoder/controller design, we assume that the receiver sends a packet acknowledgement signal ACK/NACK back to the transmitter to indicate whether it has received the packet. This ACK/NACK feedback channel between the receiver (also called the remote estimator/controller) and the transmitter/encoder is assumed to be perfect with unit delay and no packet loss. Therefore, the transmitter has exact knowledge of the packet loss sequence $\left\{\gamma_{t}\right\}$.

The remote estimator receives the intermittent sequence $\mathcal{Z}_{t} \triangleq$ $\left\{z_{0}, z_{1}, \ldots, z_{t}\right\}$, where $z_{t}=\gamma_{t}\left(s_{t}+n_{t}\right)$ based on which a control input $u_{t}$ is to be produced. We shall restrict ourselves to linear strategies. In the forthcoming section, we shall see that under perfect channel feedback (i.e., when the transmitter knows the loss sequence $\left.\Gamma_{t} \triangleq\left\{\gamma_{0}, \gamma_{1}, \ldots, \gamma_{t}\right\}\right)$, the optimal encoding strategy will be to transmit an innovation signal defined as the difference between the transmitter filtered estimate and the receiver predicted estimate. The optimal decoding/control strategy will be the cascade of a constant gain state estimator (which produces a state estimate $\bar{x}_{t \mid t}$ ) followed by a constant gain feedback from the estimated state $u_{t}:=\ell_{*} \bar{x}_{t \mid t}$. This also motivates us to consider when no channel feedback is present, a similar constant gain "estimator-controller" structure.

\section{Optimal Strategies With Quantization Compensation}

\section{A. IF (Channel Feedback)}

We now consider a general linear coder-decoder-controller scheme as follows:

1) A linear coding mechanism (with memory) produces the signal

$$
s_{t}:=\mathcal{L}_{t}\left(\mathcal{Y}_{t}, \mathcal{U}_{t-1}, \mathcal{Z}_{t-1}, \Gamma_{t-1}\right)
$$

where $\mathcal{L}_{t}\left(\mathcal{Y}_{t}, \mathcal{U}_{t-1}, \mathcal{Z}_{t-1}, \Gamma_{t-1}\right)$ is, conditionally on the packet loss sequence $\gamma_{t-1}, \ldots, \gamma_{0}$, a linear operator of its arguments $y_{t}, y_{t-1}, \ldots, y_{0}$ (the samples to be encoded), $\mathcal{U}_{t-1}=$ $\left\{u_{t-1}, \ldots, u_{0}\right\}$ and $z_{t-1}, \ldots, z_{0}$ (the past control and received signals, respectively, which can be reconstructed at the transmitter side if perfect channel feedback is available).

2) The signal $s_{t}$ is quantized and sent through a lossy channel so that the received signal $z_{t}$ can be modeled as

$$
z_{t}=\gamma_{t}\left(s_{t}+n_{t}\right)
$$

where $n_{t}$ is a white quantization noise signal with variance proportional to the variance of $s_{t}$, i.e., $\mathbb{E}\left[n_{t}^{2}\right]=\frac{\mathbb{E}\left[s_{t}^{2}\right]}{\Lambda}$. Note that the quantizer is part of the encoder in practice, so that the encoder knows the signal $s_{t}^{q}=s_{t}+n_{t}$.

3) The controller uses the received signals $z_{t}$ to build the control action at time $t$ as a (conditionally on the packet loss sequence $\gamma_{t}, \ldots, \gamma_{0}$ ) linear function $\mathcal{C}_{t}$ of the past received signals $\mathcal{Z}_{t}=$ $\left\{z_{\tau}, \tau \in[0, t]\right\}$ as well as past control signals $\mathcal{U}_{t-1}$ as

$$
u_{t}=\mathcal{C}_{t}\left(\mathcal{Z}_{t}, \mathcal{U}_{t-1}, \Gamma_{t}\right) \text {. }
$$

Note that, in principle, the conditional linear mappings $\mathcal{L}_{t}$ and $\mathcal{C}_{t}$ are time varying. The result of this section is summarized in the next proposition. The proof is deferred to the Appendix. For convenience of notation, we define

$$
\mathcal{I}_{t}^{T x}:=\left\{\mathcal{Y}_{t}, \mathcal{Z}_{t-1}, \mathcal{U}_{t-1}, \Gamma_{t-1}\right\} \quad \mathcal{I}_{t}^{R x}:=\left\{\mathcal{Z}_{t}, \mathcal{U}_{t-1}, \Gamma_{t}\right\}
$$


which are, respectively, the information sets at the transmitter and at the receiver.

Proposition 1: Consider the linear model (1) controlled through a lossy and SNR limited channel (4) using a linear encoding as in (3) and linear controller as in (5). The optimal linear quadratic strategy

$$
\left(\mathcal{C}^{*}, \mathcal{L}^{*}\right):=\underset{\mathcal{C}, \mathcal{L}}{\arg \min } \lim _{T \rightarrow \infty} \frac{1}{T} \sum_{t=0}^{T-1}\left(\mathbb{E}\left[y_{t}^{2}\right]+\rho \mathbb{E}\left[u_{t}^{2}\right]\right)
$$

satisfies the following equations:

$$
s_{t}=\mathcal{L}_{t}^{*}\left(\mathcal{I}_{t}^{T x}\right)=\hat{x}_{t \mid t}^{t x}-\bar{x}_{t \mid t-1}
$$

and

$$
u_{t}=\mathcal{C}_{t}^{*}\left(\mathcal{I}_{t}^{R x}\right)=\ell_{*} \bar{x}_{t \mid t}
$$

where $\hat{x}_{t \mid t}^{t x}=\mathbb{E}\left[x_{t} \mid \mathcal{I}_{t}^{T x}\right]$ and $\bar{x}_{t \mid t-1}=\mathbb{E}\left[x_{t} \mid \mathcal{I}_{t-1}^{R x}\right]$ can be computed as

$$
\begin{aligned}
\bar{x}_{t+1 \mid t+1} & =\left(a+\ell_{*}\right) \bar{x}_{t \mid t}+k_{I F} z_{t+1} \\
\bar{x}_{t+1 \mid t} & =\left(a+\ell_{*}\right) \bar{x}_{t \mid t} \\
\hat{x}_{t+1 \mid t+1}^{t x} & =\left(a-k_{*}\right) \hat{x}_{t \mid t}^{t x}+\ell_{*} \bar{x}_{t \mid t}+k_{*} y_{t+1}
\end{aligned}
$$

where $k_{I F}=\frac{\Lambda}{1+\Lambda}^{2}$, and the gains $\ell_{*}$ and $k_{*}$ correspond to the standard LQ controller gain and steady-state Kalman gain at the transmitter, respectively, obtained via the unique positive solutions of the Riccati equations:

$$
\begin{gathered}
m=a^{2} m+1-\frac{a^{2} m^{2}}{m+\rho}, \quad \ell_{*}=-\frac{a m}{m+\rho} \\
p=a^{2} p+\sigma_{w}^{2}-\frac{a^{2} p^{2}}{p+\sigma_{v}^{2}}, \quad k_{*}=\frac{a p}{p+\sigma_{v}^{2}} .
\end{gathered}
$$

Moreover, the optimal cost is given by

$$
J_{\infty}^{I F}=J_{\infty}\left(\mathcal{C}^{*}, \mathcal{L}^{*}\right)=\frac{\rho m}{m+\rho} \sigma_{w}^{2}+\sigma_{v}^{2}+\frac{m^{2}}{m+\rho} p_{C F}
$$

where $p_{C F}$ is the receiver predictor error covariance under IF and full channel feedback [21]; $p_{C F}$ is given by the expression

$$
p_{\mathrm{CF}}=\frac{\sigma_{w}^{2}+(1-\epsilon) \frac{\Lambda}{\Lambda+1} a^{2} p_{\infty}^{t x}}{1-a^{2} \frac{1+\epsilon \Lambda}{1+\Lambda}}
$$

where $p_{\infty}^{t x}=\frac{p-\sigma_{w}^{2}}{a^{2}}$ is the steady-state transmitter filtered state estimation error covariance. Finally, closed loop mean square stability is guaranteed if and only if the following condition is satisfied ${ }^{3}$ :

$$
\frac{1+\epsilon \Lambda}{1+\Lambda}<\frac{1}{a^{2}} \text {. }
$$

For any fixed $\epsilon$, inequality (12) can be written as

$$
\Lambda>\Lambda_{\mathrm{IF}}(\epsilon)=\frac{a^{2}-1}{\left(1-\epsilon a^{2}\right)}
$$

where $\Lambda_{\mathrm{IF}}(\epsilon)$ is the critical SQNR as a function of $\epsilon$.

Remark 1: It can be easily seen that when cheap control is considered (i.e., no control penalty term in the cost, $\rho=0$ ), $m$ in (10) satisfies $m=1$, and $J_{\infty}^{\mathrm{IF}}$ simplifies to $p_{\mathrm{CF}}+\sigma_{v}^{2}$. Note that in this case $\ell_{*}=-a$, and hence the receiver predicted state estimate $\bar{x}_{t+1 \mid t}=0 \forall t$ from (9). Thus in the cheap case, the optimal IF strategy is equivalent to state estimate forwarding at the encoder, that is, $s_{t}=\hat{x}_{t \mid t}^{t x}$ from (7) which

\footnotetext{
${ }^{2}$ See (15) in [21] for a proof of this result.

${ }^{3}$ Note, as a side that this condition is satisfied for any $\Lambda \geq 0$ and $\epsilon \in[0,1]$ if $|a|<1$.
}

is reminiscent of the strategy proposed in [19]. This observation will motivate our choice of the suboptimal SFs scheme presented below in Section IV-A.

Remark 2: The critical SQNR condition above (12) can be specialized to many existing stabilizability results for control over communication channels, such as SNR-constrained channels, simple erasure channels (with no quantization constraints) and $R$-bit erasure channels. For a comprehensive discussion on these relationships to existing stabilizability results, see [11, Sec. 5].

\section{B. Measurement Forwarding (No Channel Feedback)}

A suboptimal encoding strategy at the transmitter that does not require any channel feedback is, as proposed in [11], to simply forward the sensor measurement; namely

$$
s_{t}=y_{t}
$$

and to use a state estimator followed by a state feedback as follows:

$$
\begin{aligned}
u_{t} & =\ell \bar{x}_{t \mid t}, \quad \bar{x}_{t+1 \mid t}=a \bar{x}_{t \mid t}+u_{t} \\
\bar{x}_{t+1 \mid t+1} & =\bar{x}_{t+1 \mid t}+\gamma_{t+1} k\left(z_{t+1}-\bar{x}_{t+1 \mid t}\right)
\end{aligned}
$$

where the update step in the estimator is performed only if the packet $z_{t}$ is received. The objective is then to find the optimal gains to minimize the infinite horizon cost, i.e.,

$$
\left(k_{\mathrm{MF}}, \ell_{\mathrm{MF}}\right)=\arg \min _{k, \ell} J_{\infty} .
$$

The solution of this problem was provided in [11] in a scenario which also includes a constant transmission delay between the transmitter and the receiver. For the sake of comparison, we summarize here the main results:

Proposition 2 ([11]): The optimal gains $k_{\mathrm{MF}}, \ell_{\mathrm{MF}}$ and the corresponding optimal cost $J_{\infty}^{\mathrm{MF}}$ [see (15)] can be obtained via the solution of four coupled Riccati equations (see [11] for details). The closed loop system is mean square stable if and only if the following condition is satisfied:

$$
\frac{1+\epsilon \Lambda}{1+\Lambda}<\frac{1}{a^{2}}
$$

which is the same as (12). Therefore, the stability condition can be written as $\Lambda>\Lambda_{\mathrm{MF}}(\epsilon)=\Lambda_{\mathrm{IF}}(\epsilon)$ with the same threshold as the IF (with full channel feedback) case. In the cheap control scenario, i.e., when $\rho=0$, then $\ell_{\mathrm{MF}}=-a$ and $k_{\mathrm{MF}}=\frac{p_{\mathrm{MF}}}{p_{\mathrm{MF}}+\sigma_{v}^{2}}$, where $p_{\mathrm{MF}}>0$ is the unique positive solution of the modified Riccati equation:

$$
p_{\mathrm{MF}}=a^{2} p_{\mathrm{MF}}+\sigma_{w}^{2}-\frac{(1-\epsilon) \Lambda}{1+\Lambda} \frac{a^{2} p_{\mathrm{MF}}^{2}}{p_{\mathrm{MF}}+\sigma_{v}^{2}}
$$

and

$$
J_{\infty}^{\mathrm{MF}}=J_{\infty}\left(k_{\mathrm{MF}}, \ell_{\mathrm{MF}}\right)=\sigma_{v}^{2}+p_{\mathrm{MF}}
$$

It is important to remark that although the stability condition of the measurement forwarding strategy and the IF strategy are the same, the performance of the latter strategy is superior since

$$
J_{\infty}^{\mathrm{IF}}<J_{\infty}^{\mathrm{MF}}
$$

under any packet-loss $(\epsilon)$ and quantization $(\Lambda)$ scenarios.

\section{Suboptimal Strategies for CheAP Control Without QUANTIZATION COMPENSATION}

In this section, we consider two suboptimal strategies that design the state estimator and controller at the receiver side neglecting the pres- 
ence of quantization noise. The first scheme is based on state estimate forwarding at the encoder with the assumption of perfect ACK/NACK feedback so that the transmitter knows the exact control input, and the receiver filtering and LQ control scheme being the same as in [19]. We call it as SF with no quantization compensation at the receiver, abbreviated with SFs (SF suboptimal). The second scheme is based on measurement forwarding at the transmitter, while the receiver designs a filtering scheme ignoring any quantization noise with a constant gain (for simpler analysis) and a LQ controller as in [5]. This scheme is called measurement forwarding with no quantization compensation, abbreviated with MFs (measurement forwarding suboptimal).

Remark 3: Note that here "no quantization compensation" in SFs and MFs refers to the design of estimation and control algorithms at the receiver by neglecting quantization noise, which is nevertheless present due to the quantization in the encoding mechanism at the transmitter. The choice of these two schemes is motivated by the fact that they are schemes in existing works [19] and [5], respectively, which do not deal with rate limited channels. Finally, the results in Propositions 3 and 4 refer to the minimum SQNR required at the transmitter side (which includes the quantizer) for the cheap control case, if the no quantization compensation-based estimation and control algorithms are adopted at the receiver, as described below.

\section{A. SF With No Quantization Compensation (SFS)}

In this scheme, the state estimate [see (9)] is encoded with a finite number of bits and transmitted over the packet dropping link, so that the received signal at time $t$ is given by $\gamma_{t}\left(\hat{x}_{t \mid t}^{t x}+n_{t}\right)$. The receiver computes the state predictor $\bar{x}_{t \mid t-1}^{\mathrm{SFs}}$ :

$$
\bar{x}_{t+1 \mid t}^{\mathrm{SFs}}=a\left(\gamma_{t}\left(\hat{x}_{t \mid t}^{t x}+n_{t}\right)+\left(1-\gamma_{t}\right) \bar{x}_{t \mid t-1}^{\mathrm{SFs}}\right)+u_{t}
$$

where $u_{t}=\ell_{*}\left(\gamma_{t}\left(\hat{x}_{t \mid t}^{t x}+n_{t}\right)+\left(1-\gamma_{t}\right) \bar{x}_{t \mid t-1}^{\mathrm{SFs}}\right)$. Similarly, the transmitter predicted state estimate $\hat{x}_{t \mid t-1}^{t x}$ is given by

$$
\begin{aligned}
\hat{x}_{t+1 \mid t}^{t x}= & \left(a+\ell_{*} \gamma_{t}\right) \hat{x}_{t \mid t-1}^{t x}+\ell_{*}\left(1-\gamma_{t}\right) \bar{x}_{t \mid t-1}^{\mathrm{SFs}} \\
& +\left(a+\ell_{*} \gamma_{t}\right) k_{*}\left(\tilde{x}_{t}^{t x}+v_{t}\right)+\ell_{*} \gamma_{t} n_{t}
\end{aligned}
$$

where $\tilde{x}_{t}^{t x}=x_{t}-\hat{x}_{t \mid t}^{t x}$ is the transmitter filtering error, zero-mean Gaussian distributed with a steady-state variance of $p_{\infty}^{t x}$, as defined in Proposition 1. By defining an augmented state vector $\xi_{t}^{\mathrm{SFs}}=$ $\left[\begin{array}{ll}\hat{x}_{t \mid t-1}^{t x} & \bar{x}_{t \mid t-1}^{\mathrm{SFs}}\end{array}\right]^{\top}$ (where ${ }^{\top}$ denotes transpose), one can define the matrix $P_{t}^{\mathrm{SFs}}=\mathbb{E}\left[\xi_{t}^{\mathrm{SFs}} \xi_{t}^{\mathrm{SFs}}{ }^{\top}\right]$, and $P^{\mathrm{SFs}}=\lim _{t \rightarrow \infty} P_{t}^{\mathrm{SFs}}$. After some algebra, one can then show that $P^{\mathrm{SFs}}$ satisfies the following Lyapunov equation:

$$
P^{\mathrm{SFs}}=(1-\epsilon) \bar{A}_{1} P^{\mathrm{SFs}} \bar{A}_{1}^{\top}+\epsilon \bar{A}_{0} P^{\mathrm{SFs}} \bar{A}_{0}^{\top}+(1-\epsilon) N_{1}+\epsilon N_{0}
$$

where $\bar{A}_{1}, \bar{A}_{0}, N_{1}, N_{0}$ are given by (21) at the bottom of this page. Here $\sigma_{p}^{2}=\frac{p^{2}}{p+\sigma_{v}^{2}}$, where $p$ is given in (10), and $\left[\begin{array}{ll}1 & 0\end{array}\right] P^{\mathrm{SFs}}\left[\begin{array}{ll}1 & 0\end{array}\right]^{\top}$ is the element in position $(1,1)$ of $P^{\mathrm{SFs}}$.

For the generalized control cost with $\rho>0$, it appears to be difficult to exactly characterize when the above equation will have a unique positive definite solution. Therefore, we provide an analysis for the cheap control case, i.e., $\rho=0$. In this case, $\ell_{*}=-a$, and the above equations simplify substantially. Denoting $P^{\mathrm{SFs}}=\left[\begin{array}{cc}p_{11}^{\mathrm{SFs}} & p_{12}^{\mathrm{SFs}} \\ p_{12}^{\mathrm{SFs}} & p_{22}^{\mathrm{SFs}}\end{array}\right]$, we find that for the cheap control case, $p_{12}^{\mathrm{SFs}}=p_{22}^{\mathrm{SFs}}=0$. It can be shown that $p_{11}^{\mathrm{SFs}}$ satisfies

$$
p_{11}^{\mathrm{SFs}}=\frac{\sigma_{p}^{2}\left((1-\epsilon) \frac{a^{2}}{\Lambda}+\epsilon a^{2}\right)}{\left(1-\epsilon a^{2}\right)-\frac{a^{2}(1-\epsilon)}{\Lambda}} .
$$

It follows then that the SF scheme with no quantization compensation will have a bounded steady-state cheap control cost $\lim _{t \rightarrow \infty} \mathbb{E}\left[y_{t}^{2}\right]$, provided $\Lambda>\Lambda_{\mathrm{SFs}}(\epsilon)=\frac{a^{2}(1-\epsilon)}{1-\epsilon a^{2}}$. Or alternatively, for a fixed SQNR $\Lambda$, the maximum tolerable packet loss probability is given by $\epsilon_{\mathrm{SFs}}^{\mathrm{crit}}(\Lambda)=$ $\frac{\Lambda-a^{2}}{a^{2}(\Lambda-1)}$. Below we summarize the results of this section.

Proposition 3: Consider the SF scheme without quantization compensation given by (18), (19), assume that perfect channel feedback is available and set $\rho=0$ (cheap control). Then, the minimum SQNR required for a fixed packet loss probability is given by

$$
\Lambda_{\mathrm{SFs}}(\epsilon)=\frac{a^{2}(1-\epsilon)}{1-\epsilon a^{2}}
$$

and the corresponding (cheap) control cost is given by

$$
J_{\infty}^{\mathrm{SFs}}=\sigma_{v}^{2}+p+a^{2} \frac{p^{2}}{p+\sigma_{v}^{2}} \frac{\epsilon+\frac{1-\epsilon}{\Lambda}}{1-\epsilon a^{2}-\frac{(1-\epsilon) a^{2}}{\Lambda}}
$$

where $p$ is the transmitter prediction error variance satisfying the second equation of (10).

\section{B. MF With No Quantization Compensation}

In this scheme, the coder-decoder-controller architecture is identical to the one given in Section III-B, the only difference being the choice of the controller gain $\ell$ in (13) and of the estimator gain $k$ in (14), which in this case neglects the presence of the quantization. Therefore, these gains are chosen as:

$$
\begin{aligned}
& \left(k_{\mathrm{MFs}}, \ell_{\mathrm{MFs}}\right):=\lim _{\Lambda \rightarrow \infty} \arg \min _{k, \ell} J_{\infty} \\
& a^{2}(1-\epsilon) k_{\mathrm{MFs}}^{2}-k_{\mathrm{MFs}}\left(a^{2}-1-S_{\sigma}\right)-S_{\sigma}=0, \quad S_{\sigma}:=\frac{\sigma_{w}^{2}}{\sigma_{v}^{2}}
\end{aligned}
$$

which are exactly the gains provided in [5]. In particular, the controller gain $\ell_{\mathrm{MFs}}$ turns out to be equal to the optimal LQ gain $\ell_{*}$, while the estimator gain $k_{\mathrm{MFs}}$ satisfies the implicit equation (25). Obviously, when $\Lambda \neq 0$, the previous gains choice provides a suboptimal performance as compared to the one given by the gains given Section III-B. The performance can be derived by denoting the receiver predicted state estimate as $\bar{x}_{t \mid t-1}^{\mathrm{MFs}}$, and the corresponding prediction error as $\tilde{x}_{t}^{\mathrm{MFs}}=$

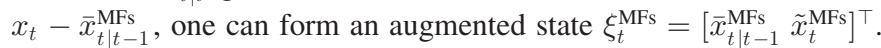
It can be shown that this augmented state vector satisfies the following

$$
\begin{aligned}
& \bar{A}_{1}=\left[\begin{array}{cc}
a+\ell_{*} & 0 \\
a+\ell_{*} & 0
\end{array}\right], \bar{A}_{0}=\left[\begin{array}{cc}
a & \ell_{*} \\
0 & a+\ell_{*}
\end{array}\right], N_{0}=\left[\begin{array}{cc}
a^{2} \sigma_{p}^{2} & 0 \\
0 & 0
\end{array}\right] \\
& N_{1}=\left[\begin{array}{cc}
\sigma_{p}^{2}\left[\left(a+\ell_{*}\right)^{2}+\frac{\ell_{L Q}^{2}}{\Lambda}\right]+\frac{\ell_{L Q}^{2}}{\Lambda}\left[\begin{array}{ll}
1 & 0
\end{array} P^{\mathrm{SFs}}\left[\begin{array}{ll}
1 & 0
\end{array}\right]^{\top}\right. & \sigma_{p}^{2}\left(a+\ell_{*}\right)^{2}+\frac{\ell_{*}\left(a+\ell_{*}\right)}{\Lambda}\left(\sigma_{p}^{2}+\left[\begin{array}{ll}
1 & 0
\end{array} P^{\mathrm{SFs}}\left[\begin{array}{ll}
1 & 0
\end{array}\right]^{\top}\right)\right. \\
\sigma_{p}^{2}\left(a+\ell_{*}\right)^{2}+\frac{\ell_{*}\left(a+\ell_{*}\right)}{\Lambda}\left(\sigma_{p}^{2}+\left[\begin{array}{ll}
1 & 0
\end{array} P^{\mathrm{SFs}}\left[\begin{array}{ll}
1 & 0
\end{array}\right]^{\top}\right)\right. & \sigma_{p}^{2}\left(a+\ell_{*}\right)^{2}\left(1+\frac{1}{\Lambda}\right)+\frac{\left(a+\ell_{*}\right)^{2}}{\Lambda}\left[\begin{array}{ll}
1 & 0
\end{array} P^{\mathrm{SFs}}\left[\begin{array}{ll}
1 & 0
\end{array}\right]^{\top}\right.
\end{array}\right] .
\end{aligned}
$$


dynamical system equations:

$$
\begin{aligned}
\xi_{t+1}^{\mathrm{MFs}}= & {\left[\begin{array}{cc}
a+\ell_{*} & \left(a+\ell_{*}\right) k_{\mathrm{MFs}} \gamma_{t} \\
0 & a\left(1-k_{\mathrm{MFs}} \gamma_{t}\right)
\end{array}\right] \xi_{t}^{\mathrm{MFs}} } \\
& +\left[\begin{array}{c}
\left(a+\ell_{*}\right) k_{\mathrm{MFs}} \gamma_{t}\left(v_{t}+n_{t}\right) \\
-a k_{\mathrm{MFs}} \gamma_{t}\left(v_{t}+n_{t}\right)+w_{t}
\end{array}\right] .
\end{aligned}
$$

Once again, defining $P_{t}^{\mathrm{MFs}}=\mathbb{E}\left[\xi_{t}^{\mathrm{MFs}} \xi_{t}^{\mathrm{MFs}}{ }^{\top}\right]$ and $P^{\mathrm{MFs}}=\lim _{t \rightarrow \infty} P_{t}^{\mathrm{MFs}}$, it can be shown that $P^{\mathrm{MFs}}$ satisfies another Lyapunov equation

$$
P^{\mathrm{MFs}}=(1-\epsilon) \tilde{A}_{1} P^{\mathrm{MFs}} \tilde{A}_{1}^{\top}+\epsilon \tilde{A}_{0} P^{\mathrm{MFs}} \tilde{A}_{0}^{\top}+(1-\epsilon) \tilde{N}_{1}+\epsilon \tilde{N}_{0}
$$

where

$$
\begin{aligned}
& \tilde{A}_{1}=\left[\begin{array}{cc}
a+\ell_{*}\left(a+\ell_{*}\right) k_{\mathrm{MFs}} \\
0 & a\left(1-k_{\mathrm{MFs}}\right)
\end{array}\right], \tilde{A}_{0}=\left[\begin{array}{cc}
a+\ell_{*} & 0 \\
0 & a
\end{array}\right] \\
& \tilde{N}_{1}=\left(\sigma_{v}^{2}+\sigma_{n}^{2}\right)\left[\begin{array}{cc}
\left(a+\ell_{*}\right)^{2} k_{\mathrm{MFs}}^{2} & -a\left(a+\ell_{*}\right) k_{\mathrm{MFs}}^{2} \\
-a\left(a+\ell_{*}\right) k_{\mathrm{MFs}}^{2} & a^{2} k_{\mathrm{MFs}}^{2}
\end{array}\right] \\
& \tilde{N}_{0}=\left[\begin{array}{cc}
0 & 0 \\
0 & \sigma_{w}^{2}
\end{array}\right] \\
& p_{22}^{\mathrm{MFs}}\left[1-a^{2}\left(1-k_{\mathrm{MFs}}\right)^{2}(1-\epsilon)-\epsilon a^{2}-\frac{(1-\epsilon) a^{2} k_{\mathrm{MFs}}^{2}}{\Lambda}\right] \\
& =\sigma_{w}^{2}+(1-\epsilon) k_{\mathrm{MFs}}^{2} \sigma_{v}^{2}\left(1+\frac{1}{\Lambda}\right) .
\end{aligned}
$$

Here, the quantization noise variance $\sigma_{n}^{2}$ is given by $\frac{1}{\Lambda}\left[\begin{array}{ll}1 & 1\end{array}\right] P^{\mathrm{MFs}}\left[\begin{array}{ll}1 & 1\end{array}\right]^{\top}+\frac{\sigma_{v}^{2}}{\Lambda}$. The optimal receiver gain $k_{\mathrm{MFs}}$ minimizes $P^{\mathrm{MFs}}(2,2)$ assuming no quantization noise $(\Lambda \rightarrow \infty)$. Once again, explicitly characterizing the set of values $(\Lambda, \epsilon)$ for which there exists a positive definite solution to (27) seems difficult for a general $\rho>0$. Below we focus on the case $\rho=0$ (cheap control) where $\ell_{*}=-a$. Denoting $P^{\mathrm{MFs}}=\left[\begin{array}{ll}p_{11}^{\mathrm{MFs}} & p_{12}^{\mathrm{MFs}} \\ p_{12}^{\mathrm{MFs}} & p_{22}^{\mathrm{MFs}}\end{array}\right]$, one can show that for $\rho=0$, $p_{11}^{\mathrm{MFs}}=p_{12}^{\mathrm{MFs}}=0$, and $p_{22}^{\mathrm{MFs}}$ satisfies (28). Exploiting this observation in the above equations, we can formulate the following proposition.

Proposition 4: For the scheme of measurement forwarding with no quantization compensation given by (26) when a cheap control cost is considered, i.e., $\rho=0$, then the minimum SQNR required for a fixed packet loss probability is given by

$$
\Lambda_{\mathrm{MFs}}(\epsilon)=\frac{a^{2}(1-\epsilon) k_{\mathrm{MFs}}^{2}}{1-\epsilon a^{2}-a^{2}(1-\epsilon)\left(1-k_{\mathrm{MFs}}\right)^{2}}
$$

and the corresponding cheap control cost is given by

$$
J_{\infty}^{\mathrm{MFs}}=\sigma_{v}^{2}+p_{22}^{\mathrm{MFs}} .
$$

Remark 4: Note that for both the above schemes, it is possible to numerically compute the control cost for any $\rho>0$, as long as there are positive definite solutions to (20) and (27). However, it is the characterization of values of $(\Lambda, \epsilon)$ for which such a solution exists that is difficult. Hence, our analysis of critical SQNR focused on the cheap control case. Later, we will illustrate the generalized control cost (for $\rho>0$ ) for these schemes via numerical simulation results.

\section{Comparison Between the Optimal and Suboptimal Strategies}

From the discussions in the previous sections, we have the following facts under any packet loss and quantization scenarios: 1) $J_{\infty}^{\mathrm{MFs}} \geq J_{\infty}^{\mathrm{MF}} \geq J_{\infty}^{\mathrm{IF}}$ and 2) $J_{\infty}^{\mathrm{SFs}} \geq J_{\infty}^{\mathrm{IF}}$. These relationships follow due to the suboptimality (ignoring quantization noise) for the schemes with

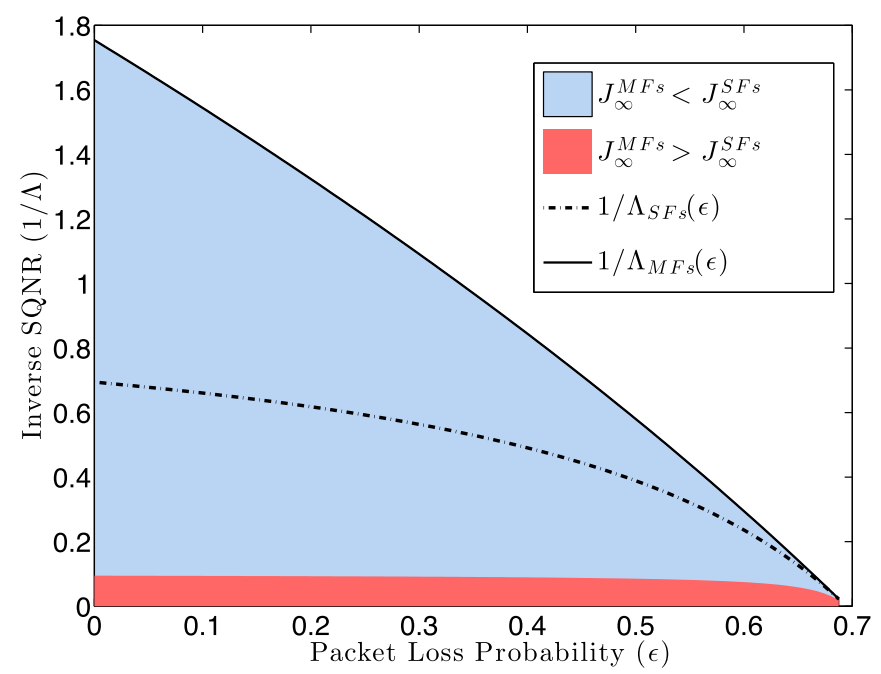

Fig. 2. Plot illustrating the regions of $(1 / \Lambda, \epsilon)$ where $J_{\infty}^{\mathrm{MFs}}<J_{\infty}^{\mathrm{SFs}}$ (blue) and vice versa (red)

no quantization compensation and the fact that IF with full channel feedback is optimal. However, it is not obvious if there is any ordering between $J_{\infty}^{\mathrm{SFs}}, J_{\infty}^{\mathrm{MFs}}$ or $J_{\infty}^{\mathrm{SFs}}, J_{\infty}^{\mathrm{MF}}$. Thus, when not compensating for quantization error, it is not obvious whether state estimate forwarding is better than measurement forwarding or vice versa; perhaps more surprisingly also the state estimate forwarding with no quantization compensation (SFs) may perform better than measurement forwarding (MF) with optimal filtering and control gains (see, e.g., Fig. 4 when $\epsilon<0.08$ ). Another interesting aspect is the relationship among the various critical SQNR (for a fixed packet loss probability $\epsilon$ ) thresholds. In particular, recall that for the optimal IF and measurement forwarding (MF), the critical SQNR is $\Lambda_{\mathrm{IF}}(\epsilon)=\Lambda_{\mathrm{MF}}(\epsilon)=\frac{a^{2}-1}{1-\epsilon a^{2}}$, and the corresponding critical SQNR for measurement forwarding with no quantization compensation is $\Lambda_{\mathrm{MFs}}(\epsilon)=\frac{a^{2}(1-\epsilon) k_{\mathrm{MFs}}^{2}}{1-\epsilon a^{2}-a^{2}(1-\epsilon)\left(1-k_{\mathrm{MFs}}\right)^{2}}$, whereas $\Lambda_{\mathrm{SFs}}(\epsilon)=\frac{a^{2}(1-\epsilon)}{1-\epsilon a^{2}}$ denotes the critical SQNR for state estimate forwarding with no quantization compensation. One can show that the following result holds whose proof is reported in the Appendix.

Proposition 5: The critical SQNRs for the various encoding schemes satisfy, for fixed $\epsilon$, the following relationship:

$$
\Lambda_{\mathrm{IF}}(\epsilon)=\Lambda_{\mathrm{MF}}(\epsilon) \leq \Lambda_{\mathrm{MFs}}(\epsilon) \leq \Lambda_{\mathrm{SFs}}(\epsilon) .
$$

Remark 5: The above result goes on to show that one requires a larger minimum SQNR for the state estimate forwarding with no quantization compensation, compared to the measurement forwarding with no quantization compensation, which in turn requires a larger SQNR threshold compared to IF/optimal measurement forwarding (which takes into account the quantization loss). While the first inequality in Proposition 5 is expected, the second inequality is somewhat counterintuitive, which is primarily due to the fact that when one does not compensate for quantization noise, closed loop control can give rise to such unexpected results.

Note also that in light of Proposition 5, one can equivalently write $\epsilon_{\mathrm{SFs}}^{\text {crit }}(\Lambda) \leq \epsilon_{\mathrm{MFs}}^{\mathrm{crit}}(\Lambda) \leq \epsilon_{\mathrm{IF}}^{\text {crit }}(\Lambda)$, where $\epsilon^{\text {crit }}$ denotes the corresponding critical packet loss probabilities for a fixed $\Lambda$.

Fig. 2 illustrates via a 2-D plot the regions on the $\left(\epsilon, \frac{1}{\Lambda}\right)$ within which MF with no quantization compensation (MFs) outperforms SF with no quantization (SFs) (blue) or vice versa (red). The parameters used for this plot are $a=1.2, \sigma_{w}^{2}=0.3, \sigma_{v}^{2}=1$. The inverse of critical SQNR 


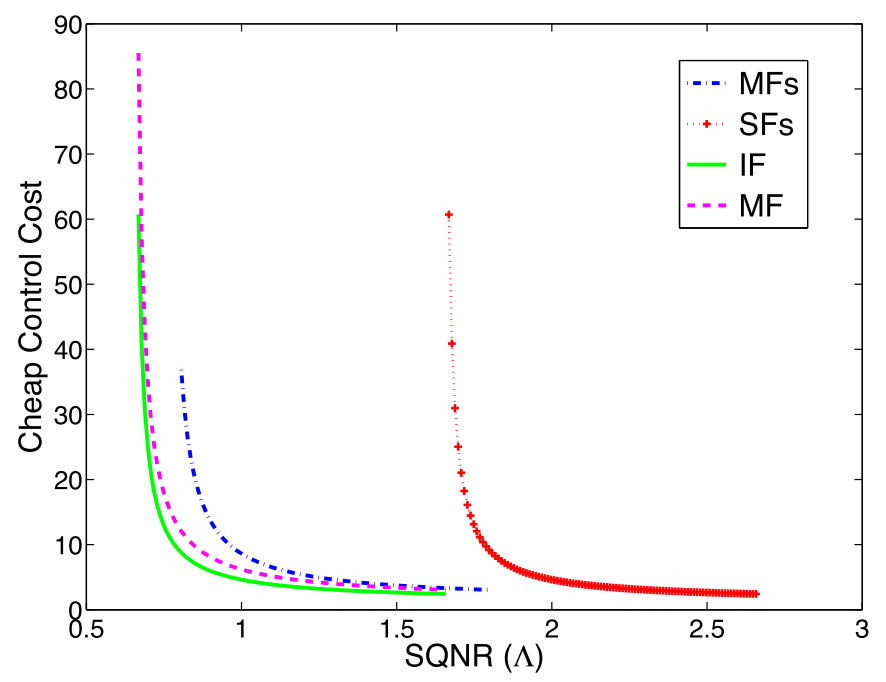

Fig. 3. Cheap control cost of optimal and suboptimal schemes versus SQNR for packet loss probability $\epsilon=0.2$.

thresholds $\frac{1}{\Lambda_{\mathrm{SFs}}(\epsilon)}$ (dash-dotted) and $\frac{1}{\Lambda_{\mathrm{MFs}}(\epsilon)}$ (solid) are also shown. It can be clearly seen that even for $\Lambda>\Lambda_{\mathrm{SFs}}(\epsilon)$ (i.e., $\left.\frac{1}{\Lambda}<\frac{1}{\Lambda_{\mathrm{SFs}}(\epsilon)}\right)$, there is a region within which $J_{\infty}^{\mathrm{MFs}}<J_{\infty}^{\mathrm{SFs}}$. More formally, we can state the following proposition:

Proposition 6: For a given $\Lambda>\Lambda_{\mathrm{SFs}}(\epsilon)$, one can find an $\epsilon^{*}(\Lambda)$ such that $J_{\infty}^{\mathrm{MFs}}<J_{\infty}^{\mathrm{SFs}}$ for $\epsilon>\epsilon^{*}(\Lambda)$, and similarly, for a given $\epsilon$, one can find a $\Lambda^{*}(\epsilon)$ such that for $\Lambda<\Lambda^{*}(\epsilon), J_{\infty}^{\mathrm{MFs}}<J_{\infty}^{\mathrm{SFs}}$.

The proof of this result is elementary and is omitted.

\section{NumERical REsults}

In this section, we present some numerical results on a comparative study of the various encoder-decoder design strategies discussed in the earlier sections. We first consider a linear dynamical system with $a=1.2$, and $\sigma_{w}^{2}=0.3, \sigma_{v}^{2}=1.0$.

In Fig. 3, we plot the cheap control cost $(\rho=0)$ for a fixed packet loss probability $(\epsilon=0.2)$ for the two optimal schemes against the SQNR $\Lambda$ : IF with full channel feedback and measurement forwarding with optimal filtering and control gains, and the two suboptimal schemes: SF with no quantization compensation (SFs) and MF with no quantization compensation (MFs). The effect of the different SQNR thresholds are clearly visible in that the control cost rises sharply as $\Lambda$ approaches these thresholds. In particular, for this example, $\Lambda_{\mathrm{IF}}(0.2)=$ $\Lambda_{\mathrm{MF}}(0.2)=0.6180, \Lambda_{\mathrm{MFs}}(0.2)=0.7555$, and $\Lambda_{\mathrm{SFs}}(\epsilon)=1.6180$.

Next, we consider a linear dynamical system with $a=1.2, \sigma_{w}^{2}=$ $0.1, \sigma_{v}^{2}=0.5$, and a signal-to-noise ratio $\Lambda:=1.6875$ (corresponding to a 2-bits quantizer). We compare, as a function of the packet loss probability $\epsilon$, the performance of the same schemes (optimal and suboptimal ones) both for the cheap ( $\rho=0)$ as well as noncheap $(\rho=2)$ scenarios. The results are reported in Fig. 4. It is clearly seen that the IF scheme provides the best performance, while the MF and MFs schemes offer close performance with MFs performing a little worse for not compensating for quantization noise. In the cheap control case, the SF with no quantization compensation (SFs) performs much worse than the MFs scheme for all packet loss probabilities. In the noncheap case, it is seen that SFs performs marginally better than MFs at low packet loss probabilities, but as packet loss probability increases, it quickly becomes worse than MFs ( at around approximately $\epsilon=0.15$ for $\rho=2$ ), since the critical probability for SFs is lower than that of
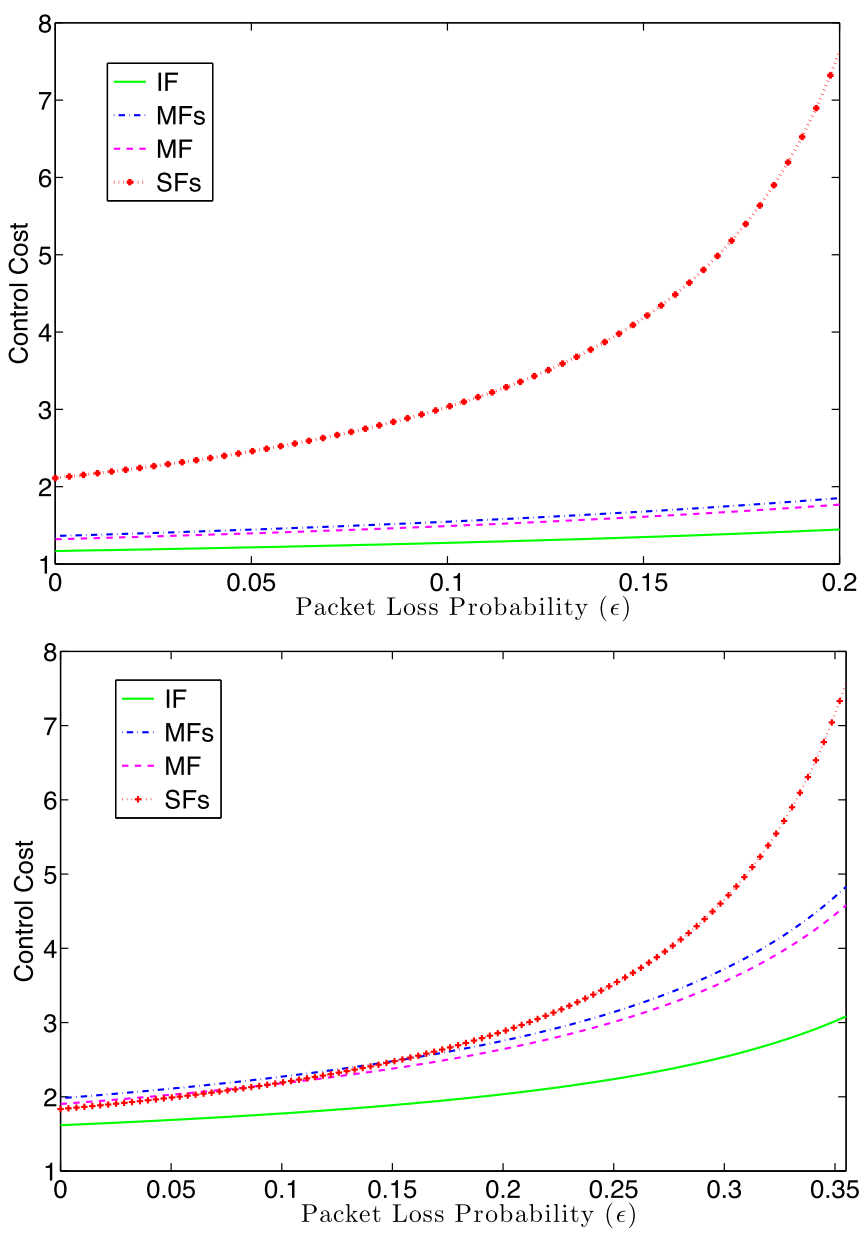

Fig. 4. Control cost $J_{\infty}$ of optimal and suboptimal schemes versus packet loss probability for the cheap control scenario $\rho=0$ (top panel), and for $\rho=2$ (bottom panel) for $\Lambda=1.6875$ (2-bit quantizer).

MFs. The exact value of this crossover point is difficult to determine analytically for a given $\rho$, as it is given by a solution of higher degree nonlinear equation.

\section{CONCLUSION}

In this paper, we addressed the problem of closed loop optimal control in the presence of limited capacity channels modeled via the inclusion of packet loss and quantization. We have shown that the optimal linear coder-decoder-controller strategy (IF) under the assumption of perfect channel feedback for the transmitter, i.e., under lossless ACK/NACK channel, corresponds to transmitting the quantized difference between the best state estimate at the transmitter and the predicted state estimate at the receiver, reminiscent of the DPCM scheme adopted in lossless communication protocols. Although the stability region in terms of feasible packet loss probability and SQNR is not enlarged as compared to a strategy that simply forwards the measurements without any preprocessing, the control performance is greatly improved. We also studied two suboptimal strategies which are popular strategies in the existing literature, but do not account for quantization, based on state estimate forwarding (SFs) and measurement forwarding (MFs). In this suboptimal scenario, it is shown that, surprisingly, the suboptimal MF strategy (MFs) is always superior to suboptimal SF strategy (SFs) for small SQNR values. Future research directions include the analysis 
for vector dynamical systems, channels with memory such as Markovian channels, and lossy channel feedback, i.e., when the ACK/NACK packets are subject to losses as well. Inspired by results such as in [26], [27], and [28], we will also investigate the existence of possible nonlinear controllers that outperform linear controllers in the case of such imperfect acknowledgements.

\section{APPENDIX}

Proof of Proposition 1: First of all, we consider a finite horizon version of the optimal control problem (6)

$$
\begin{aligned}
& J_{T}^{*}=\min _{\mathcal{C}, \mathcal{L}} J_{T} \\
& J_{T}:=\frac{1}{T} \sum_{k=0}^{T-1} \mathbb{E}\left[y_{k}^{2}+\rho u_{k}^{2}\right]=\sigma_{v}^{2}+\frac{1}{T} \sum_{k=0}^{T-1} \mathbb{E}\left[x_{k}^{2}+\rho u_{k}^{2}\right]
\end{aligned}
$$

where the expectation $\mathbb{E}[\cdot]$ is performed not only over the measurement, process, and quantization noise processes, but also across the packet loss process.

Let us consider the optimal control design as a function of any fixed (but possibly time-varying) coding scheme $\mathcal{L}:=\left\{\mathcal{L}_{k}, k \in[0, T)\right\}$. We define the corresponding cost-to-go function as

$$
\begin{aligned}
V_{k}^{\mathcal{L}}=V_{k}^{\mathcal{L}}\left(\mathcal{I}_{k}^{R x}\right) & :=\min _{u_{k}=\mathcal{C}_{k}\left(\mathcal{I}_{k}^{R x}\right)} G_{k}^{\mathcal{L}}\left(\mathcal{I}_{k}^{R x}, u_{k}\right) \\
G_{k}^{\mathcal{L}}\left(\mathcal{I}_{k}^{R x}, u_{k}\right) & =\mathbb{E}\left[x_{k}^{2}+\rho u_{k}^{2}+V_{k+1}^{\mathcal{L}}\left(\mathcal{I}_{k+1}^{R x}\right) \mid \mathcal{I}_{k}^{R x}\right] .
\end{aligned}
$$

Let us further define

$$
\begin{aligned}
& \bar{x}_{k \mid h}:=\mathbb{E}\left[x_{k} \mid \mathcal{I}_{h}^{R x}\right], p_{k \mid h}:=\operatorname{Var}\left\{x_{k}-\bar{x}_{k \mid h} \mid \mathcal{I}_{h}^{R x}\right\} \\
& \bar{p}_{k \mid h}:=\mathbb{E}\left[p_{k \mid h}\right] .
\end{aligned}
$$

We claim that the optimal cost-to-go function can be written as

$$
V_{k}^{\mathcal{L}}=m_{k} \mathbb{E}\left[x_{k}^{2} \mid \mathcal{I}_{k}^{R x}\right]+c_{k}
$$

where $m_{k}$ is a constant while $c_{k}$ is $\mathcal{I}_{k}^{R x}$ - measurable. We prove this by induction. The expression in clearly true for $k=T$ with $c_{T}=m_{T}=0$. Assume that the expression is true for $k+1$ and let us prove it for $k$ :

$$
\begin{aligned}
G_{k}^{\mathcal{L}}\left(\mathcal{I}_{k}^{R x}, u_{k}\right)= & \mathbb{E}\left[x_{k}^{2}+\rho u_{t}^{2}+m_{k+1} \mathbb{E}\left[x_{t+1}^{2} \mid \mathcal{I}_{k+1}^{R x}\right]+c_{k+1} \mid \mathcal{I}_{k}^{R x}\right] \\
= & \mathbb{E}\left[x_{k}^{2}+\rho u_{t}^{2}+m_{k+1}\left(a x_{t}+u_{t}+w_{t}\right)^{2}\right. \\
& \left.+c_{k+1} \mid \mathcal{I}_{k}^{R x}\right] \\
= & \left(a^{2} m_{k+1}+1\right) \mathbb{E}\left[x_{k}^{2} \mid \mathcal{I}_{k}^{R x}\right]+\left(\rho+m_{k+1}\right) u_{t}^{2} \\
& +m_{k+1}\left(\sigma_{w}^{2}+2 a \bar{x}_{k \mid k} u_{k}\right)+\mathbb{E}\left[c_{k+1} \mid \mathcal{I}_{k}^{R x}\right]
\end{aligned}
$$

therefore we get

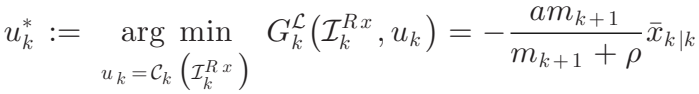

$$
\begin{aligned}
& V_{k}^{\mathcal{L}}=\left(a^{2} m_{k+1}+1-\frac{a^{2} m_{k+1}^{2}}{m_{k+1}+\rho}\right) \mathbb{E}\left[x_{k}^{2} \mid \mathcal{I}_{k}^{R x}\right] \\
& +\frac{a^{2} m_{k+1}^{2}}{m_{k+1}+\rho} p_{k \mid k}+m_{k+1} \sigma_{w}^{2}+\mathbb{E}\left[c_{k+1} \mid \mathcal{I}_{k}^{R x}\right]
\end{aligned}
$$

from which it follows that:

$$
\begin{aligned}
m_{k} & :=a^{2} m_{k+1}+1-\frac{a^{2} m_{k+1}^{2}}{m_{k+1}+\rho} \\
c_{k} & :=\mathbb{E}\left[c_{k+1}+m_{k+1} \sigma_{w}^{2}+\frac{a^{2} m_{k+1}^{2}}{m_{k+1}+\rho} p_{k \mid k} \mid \mathcal{I}_{k}^{R x}\right] .
\end{aligned}
$$

Therefore, the total cost for a fixed encoding strategy is given by:

$$
\begin{aligned}
J_{T}^{\mathcal{L}} & =\min _{\mathcal{C}} J_{T} \\
& =\frac{v_{0}}{T} \mathbb{E}\left[x_{0}^{2}\right]+\frac{1}{T} \sum_{k=1}^{T} m_{k} \sigma_{w}^{2}+\frac{a^{2} m_{k}^{2}}{m_{k}+\rho} p_{k-1 \mid k-1} \\
& =\frac{v_{0}}{T} \mathbb{E}\left[x_{0}^{2}\right]+\frac{1}{T} \sum_{k=1}^{T}\left(\frac{\rho m_{k}}{m_{k}+\rho} \sigma_{w}^{2}+\frac{m_{k}^{2}}{m_{k}+\rho} p_{k \mid k-1}\right)
\end{aligned}
$$

where we used the fact that $p_{k+1 \mid k}=a^{2} p_{k \mid k}+\sigma_{w}^{2}$. What is left to do is to find the optimal encoding strategy which minimizes $J_{\mathcal{T}}^{\mathcal{L}}$. For full channel feedback the transmitter knows the applied input signal, therefore the expected estimation error variances $p_{k \mid k} \mathrm{~s}$ do not depend on the control input $\left(u_{0}, \ldots, u_{T-1}\right)$. Moreover, since the cost function is a linear combination of these expected estimation error variances with positive coefficient and each $p_{k+1 \mid k+1}$ is monotonically increasing function of the previous value $p_{k \mid k}$, then the optimal encoding strategy is the one that minimizes $p_{k \mid k}$ for each $k$. In [21], it has been shown, in the context of remote estimation, that the optimal encoding strategy is to transmit the innovation, i.e., the difference between the optimal estimate of the state at the transmitter and the optimal one-step prediction of the same state at the receiver. The same proof can be carried along in this context since $p_{k \mid k}$ does not depend on the input sequence thus leading to exactly the same expression as in [21]:

$$
p_{k+1 \mid k}=a^{2} p_{k \mid k-1}+\sigma_{w}^{2}-(1-\epsilon) \frac{a^{2} \Lambda}{1+\Lambda}\left(p_{k \mid k-1}-p_{k \mid k}^{t x}\right)
$$

where $p_{k \mid k}^{t x}=\operatorname{Var}\left\{x_{k}-x_{k \mid k}^{t x}\right\}$. If we now consider the infinite horizon problem by taking the limit $T \rightarrow \infty$ we obtain the optimal cost given in (11) where

$$
p^{\mathrm{CF}}=\lim _{k \rightarrow+\infty} p_{k+1 \mid k}, \quad m=\lim _{T \rightarrow+\infty} m_{0}, p_{\infty}^{t x}=\lim _{k \rightarrow+\infty} p_{k \mid k}^{t x} .
$$

Proof of Proposition 5: By using the expression for $\Lambda_{\mathrm{SFs}}(\epsilon)$ and $\Lambda_{\mathrm{MFs}}(\epsilon)$, it is easy to show that

$$
\Lambda_{\mathrm{MFs}}(\epsilon)=\frac{\Lambda_{\mathrm{SFs}}(\epsilon) k_{\mathrm{MFs}}^{2}}{1-\Lambda_{\mathrm{SFs}}(\epsilon)\left(1-k_{\mathrm{MFs}}\right)^{2}} .
$$

Using the first equation of (28), it is easy to show that $1-\Lambda_{\mathrm{SFs}}(\epsilon)(1-$ $\left.k_{\mathrm{MFs}}\right) \geq 0$. Also, it is easy to show that

$$
\frac{\partial k_{\mathrm{MFs}}}{\partial S_{\sigma}}>0, \lim _{S_{\sigma} \rightarrow 0} k_{\mathrm{MFs}}=k_{\mathrm{min}}=\frac{a^{2}-1}{a^{2}-\epsilon a^{2}}, \lim _{S_{\sigma} \rightarrow \infty} k_{\mathrm{MFs}}=1 .
$$

Next, after some algebraic manipulations, it follows that $\frac{\partial \Lambda_{\mathrm{MFs}}(\epsilon)}{\partial k_{\mathrm{MFs}}} \geq$ 0 for $k_{\mathrm{min}} \leq k_{\mathrm{MFs}} \leq 1$. One can now use (32), and show that the minimum and maximum values of $\Lambda_{\mathrm{MFs}}(\epsilon)$ can be obtained by substituting $k_{\mathrm{MFs}}=k_{\mathrm{min}}$ and $k_{\mathrm{MFs}}=1$, respectively, yielding $\Lambda_{\mathrm{MFs}}^{\mathrm{min}}=\Lambda_{I F}(\epsilon)$ and $\Lambda_{\mathrm{MFs}}^{\max }=\Lambda_{\mathrm{SFs}}(\epsilon)$. The first relationship follows by noting that $k_{\mathrm{min}}=1-\frac{1}{\Lambda_{\mathrm{SFs}}(\epsilon)}$, and $\Lambda_{\mathrm{IF}}(\epsilon)=\Lambda_{\mathrm{SFs}}(\epsilon)-1$. Hence for a fixed $\epsilon$, and any $0<S_{\sigma}<\infty, \Lambda_{\mathrm{IF}}(\epsilon)<\Lambda_{\mathrm{MFs}}(\epsilon)<\Lambda_{\mathrm{SFs}}(\epsilon)$ with the inequalities being tight at the boundary values of $S_{\sigma}=0$ and $S_{\sigma} \rightarrow \infty$, respectively.

\section{REFERENCES}

[1] J. H. Braslavsky, R. H. Middleton, and J. S. Freudenberg, "Feedback stabilization over signal-to-noise ratio constrained channels," in Proc. Amer. Control Conf., Jun. 2004, pp. 4903-4908.

[2] S. Tatikonda and S. Mitter, "Control under communication constraints," IEEE Trans. Autom. Control, vol. 49, no. 7, pp. 1056-1068, Jul. 2004. 
[3] A. Sahai and S. Mitter, "The necessity and sufficiency of anytime capacity for control over a noisy communication link: Part I," IEEE Trans. Inf. Theory, vol. 52, no. 8, pp. 3369-3395, Aug. 2006.

[4] N. Elia and J. Eisembeis, "Limitation of linear control over packet drop networks," in Proc. IEEE Conf. Decis. Control, vol. 5, Dec. 2004, pp. $5152-$ 5157.

[5] L. Schenato, B. Sinopoli, M. Franceschetti, K. Poolla, and S. Sastry, "Foundations of control and estimation over lossy networks," Proc. IEEE, vol. 95, no. 1, pp. 163-187, Jan. 2007.

[6] N. C. Martins and M. A. Dahleh, "Feedback control in the presence of noisy channels: "Bode-like" fundamental limitations of performance," IEEE Trans. Autom. Control, vol. 53, no. 7, pp. 1604-1615, Aug. 2008.

[7] A. Matveev and A. Savkin, "The problem of LQG optimal control via a limited capacity communication channel," Syst. Control Lett., vol. 53, no. 1, pp. 51-64, 2004.

[8] K. Tsumura, H. Ishii, and H. Hoshina, "Tradeoffs between quantization and packet loss in networked control of linear systems," Automatica, vol. 45, no. 12, pp. 2963-2970, 2009.

[9] P. Minero, L. Coviello, and M. Franceschetti, "Stabilization over Markov feedback channels: The general case," Trans. Autom. Control, vol. 58, no. 2, pp. 349-362, 2013.

[10] E. Silva and S. Pulgar, "Performance limitations for single-input LTI plants controlled over SNR constrained channels with feedback," Automatica, vol. 2, no. 49, pp. 540-547, 2013.

[11] A. Chiuso, N. Laurenti, L. Schenato, and A. Zanella, "LQG-like control of scalar systems over communication channels: The role of data losses, delays and SNR limitations," Automatica, vol. 50, no. 12, pp. 3155-3163, 2014.

[12] V. Borkar and S. Mitter, "LQG control with communication constraints," in Festschrift: Communications, Computation, Control and Signal Processing: A Tribute to Thomas Kailath. Norwell, MA, USA: Kluwer, 1997.

[13] O. C. Imer, S. Yuksel, and T. Basar, "Optimal control of dynamical systems over unreliable communication links," Automatica, vol. 42, no. 9, pp. 1429-1440, Sep. 2006.

[14] B. Sinopoli, L. Schenato, M. Franceschetti, K. Poolla, and S. Sastry, “Optimal linear LQG control over lossy networks without packet acknowledgment," Asian J. Control, vol. 10, pp. 3-13, 2008.
[15] M. Trivellato and N. Benvenuto, "State control in networked control systems under packet drops and limited transmission bandwidth," IEEE Trans. Commun., vol. 58, no. 2, pp. 611-622, Feb. 2010.

[16] E. Garone, B. Sinopoli, and A. Casavola, "LQG control over lossy TCP like networks with probabilistic packet acknowledgment," Int. J. Syst. Control Commun., vol. 2, pp. 55-81, 2010.

[17] M. Epstein, L. Shi, and R. M. Murray, "Estimation schemes for networked control systems using UDP-like communication," in Proc. IEEE Conf. Decis. Control, Kos, Greece, 2007, pp. 3945-3951.

[18] S. Dey, A. Chiuso, and L. Schenato, "Linear encoder-decoder-controller design over channels with packet loss and quantization noise," in Proc. Eur. Conf. Control, Jul. 2015, pp. 934-939.

[19] V. Gupta, D. Spanos, B. Hassibi, and R. M. Murray, "Optimal LQG control across a packet-dropping link," Syst. Control Lett., vol. 56, no. 6 , pp. 439-446, 2007.

[20] S. Haykin, Communication Systems, John Wiley and Sons, New York, 2001.

[21] S. Dey, A. Chiuso, and L. Schenato, "Remote estimation with noisy measurements subject to packet loss and quantization noise," IEEE Trans. Control Netw. Syst., vol. 1, no. 3, pp. 204-217, Sep. 2013.

[22] K. You and L. Xie, "Minimum data rate for mean square stabilizability of linear systems with Markovian packet losses," IEEE Trans. Autom. Control, vol. 56, no. 4, pp. 772-785, Apr. 2011.

[23] D. Marco and D. Neuhoff, "The validity of the additive noise model for uniform scalar quantizers," IEEE Trans. Inf. Theory, vol. 51, no. 5, pp. 1739-1755, May 2005.

[24] V. Goyal, "High-rate transform coding: How high is high, and does it matter?" in Proc. IEEE Int. Symp. Inf. Theory, 2000, p. 207.

[25] A. Leong, S. Dey, and G. Nair, "Quantized filtering schemes for multisensor linear state estimation: Stability and performance under high rate quantization," IEEE Trans. Signal Process., vol. 61, no. 15, pp. $3852-$ 3865, Aug. 2013.

[26] H. S. Witsenhausen, "A counterexample in stochastic optimal control," SIAM J. Control, no. 1, pp. 131-147, Jan. 1968.

[27] M. Andersson, A. A. Zaidi, N. Wernersson, and M. Skoglund, "Nonlinear distributed sensing for closed-loop control over Gaussian channels," in Proc. IEEE Swedish Commun. Technol. Workshop, 2011, pp. 19-23.

[28] S. Yüksel and S. Tatikonda, "A counterexample in distributed optimal sensing and control," IEEE Trans. Autom. Control, vol. 54, no. 4, pp. 841844, Apr. 2009. 